\title{
ANÁLISIS VIVENCIAL DE LA INTERACCIÓN EDUCATIVA PARA LA DETERMINACIÓN DE CONDICIONES ESPACIALES DE AULA EN URUGUAY
}

Paula Cardellino 


\section{PAULA CARDELLINO}

Doctora en Arquitectura y Urbanismo, Universidad del Bío Bío (Chile). Master Intelligent Buildings, University of Reading (Reino Unido). Docente e Investigadora, Facultad de Arquitectura, Universidad ORT Uruguay. Investigadora Nivel I, Sistema Nacional de Investigadores (SNI), Agencia Nacional de Investigación e Innovación (Uruguay).

FECHA DE RECEPCIÓN: 27 de agosto de 2018

FECHA DE ACEPTACIÓN: 14 de noviembre de 2018.

REGISTRO BIBLIOGRÁFICO: Cardellino, P. (2018). Análisis vivencial de la interacción educativa para la determinación de condiciones espaciales de aula en Uruguay. Anales de Investigación en Arquitectura, 8, 25-42. 


\section{RESUMEN}

La arquitectura educacional es un soporte crucial para lograr educación de calidad. Sin embargo, su diseño actual presenta una uniformidad formal basada en condiciones constructivas de hace un siglo con escasas referencias a cambios sociales y particularidades funcionales. Esto es notorio en el espacio de aula donde forma y dimensión son parámetros incuestionables por parte de los responsables de su diseño como por aquellos que lo usan. Este artículo presenta una metodología de análisis vivencial aplicada al aula escolar para diagnosticar la relación alumno - maestro que permita determinar características arquitectónicas que contribuyan a una mejor interacción. En aulas uruguayas se analizan: información fotográfica obtenida in situ, registros video-fotográficos, y entrevistas con maestras. Las conclusiones señalan que este corpus metodológico permite cuantificar variaciones perceptivas según relaciones de proporción y distribución espacial. Se enfatiza también la necesidad de integrar registros perceptivos al diseño arquitectónico para asegurar la calidad de las relaciones.

Palabras Clave: Escuelas; aula escolar; interacción alumno - maestro; diseño arquitectónico; análisis vivencial.

\section{ABSTRACT}

Educational architecture is crucial to support quality education. However, its current design presents a formal uniformity based on constructive conditions 200 years old with few references to social changes and functional characteristics. This is evident in the classroom space where its shape and dimension are considered to be unquestioned by those responsible for its design and those who use it. This paper presents a methodology of experiential analysis applied to the classroom environment to diagnose pupils -teacher relationship to determine architectural characteristics that can contribute to a better interaction. In Uruguayan classrooms photographic information obtained in situ, video-photographic records and interviews with teachers are analyzed. The findings suggest that this methodological corpus can quantify perceptual variations related to proportion and spatial distribution. The need to integrate perceptual records to architectural design to ensure the quality of relationships is also emphasized.

Keywords: Schools, classroom; interaction pupil-teacher; experiential analysis; architectural design. 


\section{INTRODUCCIÓN}

La infraestructura educacional se considera crucial para lograr una educación de calidad (Horne-Martin, 2004; Higgins et al., 2005; Woolner, 2010; Darmody y Smyth, 2012). Sin embargo, el diseño de los establecimientos actuales, en su mayoría, presentan una uniformidad formal basada en condiciones constructivas que datan de hace más de un siglo con escasas referencias a los cambios sociales, particularidades funcionales $\mathrm{y} / \mathrm{o}$ culturales del lugar y del momento (Burgos, 2001; Gislason, 2007; Leiringer y Cardellino, 2011; Woolner, 2015). Esto es particularmente notable en el aula escolar (Scott-Webber et al., 2014; Barrett et al., 2015). Si bien existen estudios sobre la transformación del espacio de aula en general (Dussel y Carusso, 1999; Cabanellas y Eslava, 2005), desde el punto de vista pedagógico (Brailovsky, 2013) y el arquitectónico (Grementieri y Shmidt, 2010), así como para el caso de Uruguay en particular (Barran, 2008), el aula tradicionalmente concebida ha permanecido prácticamente inalterada en su proporción y distribución espacial. El aula suele ser tomada como incuestionable por parte de los responsables del diseño de los contenedores educativos e inalterable por aquellos que lo usan (Cardellino et al., 2017).

La forma rectangular de aula, con bancos y sillas dispuestas en filas rectas y con amplias ventanas, resulta legítima y necesaria a comienzos del siglo XX ya que satisface las necesidades básicas del momento. El aula estrecha y larga, por ejemplo, surge de la necesidad de proveer iluminación a todo el espacio, previo a la aparición de la luz eléctrica. El frente de la clase quedaba, entonces, determinado por la localización de las ventanas, ya que los alumnos se sentaban de forma que la luz proveniente de la ventana ingresara por encima de su hombro izquierdo (Sommer, 2007). A pesar de cambios y desarrollos en aspectos lumínicos, acústicos y de estructura, la arquitectura escolar continúa estando compuesta por 'cajas' que contienen aulas, que se caracterizan por su forma rectangular y por la disposición tradicional del mobiliario donde los alumnos se sientan en filas con la maestra al frente de la clase constituyendo la fuente principal de información junto al pizarrón (Lim et al., 2012). Si bien existen ejemplos que intentan romper con el patrón rígido y estructurado (por ejemplo: Burgos, 2001; Barran, 2008; Hertzberger, 2008) y aunque los primeros investigadores en educación promovieron de forma invariable un aprendizaje basado en una inmersión en el mundo o sociedad y no solo en el aula (por ejemplo: Rousseau, Pestalozzi, Fröebel y Steiner), las escuelas consideradas "experimentales" resultan ser la excepción y no la regla.

Recientemente, se dispone de nuevos medios de registro y estrategias de análisis perceptivo vivencial que demuestran aportes en estudios de corte antropológico y urbano que, aplicados 
a la arquitectura de pequeña escala, resultan útiles para diagnosticar el desempeño actual de los espacios escolares e identificar condiciones de diseño que contribuyan a su mejor utilización en el futuro. En este sentido, la aplicación de estos medios ofrece la oportunidad de obtener una nueva perspectiva sobre la relación espacio/usuario y el espacio.

En este artículo se presenta una metodología de análisis vivencial de la interacción educativa para determinar condicionantes arquitectónicas del aula. Se toma como punto de partida el concepto de aula como contenedor formal donde ocurre la interacción educativa entre maestro y alumnos y donde el fenómeno de enseñanza y aprendizaje se define como el proceso de comunicación directa, cara a cara, entre el maestro y los alumnos. De acuerdo con la teoría de la comunicación, para que la recepción de un mensaje sea óptima ésta debe llegar al receptor sin ruido que lo distorsione en el camino (Davis, 1978). Esta interacción se lleva a cabo en un espacio determinado, el aula, durante períodos definidos con tradiciones culturales propias y en organizaciones institucionales masificadas. De esta forma, el estudio se basa en un estudio de casos de aulas tradicionales en Uruguay donde se le presta especial atención a la relación entre las interacciones educativas maestro - alumno y las condiciones espaciales de las aulas. Se concluye que hay aspectos espaciales de proporción del aula y de disposición espacial del mobiliario que afectan los registros perceptivos de los alumnos.

\section{LAS PERSONAS COMO OBJETO DE ESTUDIO}

Esta forma de investigar deriva del movimiento científico/filosófico fenomenológico que surge de manera oficial con el filósofo alemán Edmund Husserl (1859 - 1938) y que aboga por el estudio sin supuestos previos (prejuicios o hipótesis) de los fenómenos apelando a la experiencia o percepción pura, la que bien practicada nos aproxima a la esencia de los objetos observados. Sin embargo, es Goethe quien señala que la fenomenología no implica separar al observador del fenómeno, sino que busca un encuentro íntimo con el fenómeno a través de los sentidos educables de la percepción humana (Seamon y Zajonc, 1998). Por tanto, el énfasis que Goethe es el de la permanencia o fidelidad del observador con la experiencia del fenómeno durante el transcurso del estudio. Este es un punto de contraste crucial con el estilo fenomenológico de Husserl donde, aunque el investigador comienza con la experiencia del fenómeno, luego retrocede y lo examina cerebralmente a través de la reflexión y otras herramientas del intelecto (Varela et al., 2009).

Para la fenomenología goetheana la teoría surge de la experiencia misma, del estado contemplativo del objeto donde la clave de la comprensión del mundo depende de la copresencia y codependencia entre objeto y sujeto (del Solar, 1993). En este sentido, varios autores argumentan que la fenomenología de 
Goethe no ha sido aún superada (Steiner, 1989, 2000, 2008; Seamon y Zajonc, 1998; Zajonc, 1994; Amrine et al., 1987; Amrine, 1995).

El foco central de la fenomenología en el quehacer arquitectónico tiene que ver con la forma en que las personas existen en relación con su mundo. Heidegger (1962) argumenta que la relación entre las personas y su mundo es indivisible ya que la persona no puede existir aparte de su mundo, sino que ambas están íntimamente relacionadas. Stewart y Mickunas (1990) las consideran como una unidad indisoluble y Heidegger (1962) como being-inthe-world (ser en el mundo). De este modo, no es posible disociar a la persona del mundo, y viceversa, ya que ambos existen siempre unidos $y$, por lo tanto, solo pueden ser interpretados en términos de una relación holística (Seamon, 1990; 2000). Entonces, uno de los desafíos fenomenológicos es la descripción de esta íntima relación persona-mundo de manera que legítimamente escapen de cualquier dicotomía sujeto-objeto (Seamon, 2000).

Esto dicho, esta rama de investigación centrada en el estudio del comportamiento humano ha permanecido, en cierta forma, en un estado de subdesarrollo metodológico. Su instrumentalización hasta la fecha ha sido exigua y dispersa. Una de las razones por las cuales ha quedado, de alguna forma, rezagada es que, en general, los autores se han remitido a hablar de la teoría sin sugerir, o testear, una posible metodología para su análisis. Este subdesarrollo metodológico tiene repercusiones a la hora de proyectar complejos arquitectónicos ya que las consideraciones de aspectos vivenciales no son tomadas en cuenta (Helio Piñon, 2006; Muñoz Cosme, 2008; Corona Martínez, 2009). Los diseños arquitectónicos tienden a estar basados en programas arquitectónicos preestablecidos y de "supuestos" vivenciales - es decir, de la memoria personal de las actividades.

Desde los estudios seminales de Hall (1969) concernientes con el impacto que el espacio tiene sobre el comportamiento humano se sabe que el espacio hecho por el hombre influye directamente en su comportamiento así como en las relaciones humanas que dentro de él se desarrollan (Hall, 1969). Esto, a su vez, impacta en la forma en que se organiza el espacio circundante. Naturalmente, la mejor manera de testear un espacio es experimentar personalmente la relación espacial y la escala del lugar. Una vez que se comienza a medir, reunir y sistematizar las observaciones personales, los conceptos como escala humana, sentidos y necesidades toman un significado más concreto. Estos conceptos ya no son incorporados como una idea luego de finalizado el proyecto, sino se incorporan naturalmente desde el comienzo del diseño para las 'personas' (Lawson, 2001; Gehl y Svarre, 2013). 


\section{EL AULA ESCOLAR COMO ESCENARIO DE INTERACCIÓN}

Maestros y alumnos pasan la mayor parte de su día dentro del aula donde mantienen una relación educativa formal. En este sentido, el aula es considerada como espacio social de participación e interacción dentro del ámbito escolar (Fragoso Franco, 1999). Esto le provee al aula un estatus único entre los espacios de uso humano, considerado entre las estructuras físicas más importantes de la sociedad. El entorno físico del aula puede estimular o inhibir el tipo de interacción deseada, y por lo tanto el aprendizaje (García Ponce, 2000). Por ejemplo, la distribución espacial de los alumnos determina su mayor o menor participación, que a su vez depende del tamaño del aula y de si se ubican o no en el campo visual del maestro. La capacidad de "hacer contacto visual" (mirar a alguien a los ojos) es, al menos en la cultura occidental, la clave para establecer la comunicación en un grupo, y es particularmente importante para el maestro.

El estudio del aula como escenario de interacción físicamente estructurado ha sido limitado. En efecto, Marx y colegas (2000) afirman que entre los aspectos físicos del aula que necesitan consideración son aquellos que dictaminan la posición relativa de los alumnos en relación con el maestro. Estudios sobre la distribución del mobiliario en este espacio suelen coincidir en que la distancia entre el maestro y los alumnos influye en la calidad de la interacción y la comunicación (Holliman y Anderson, 1986; Gump, 1987).Varios estudios argumentan que la interacción educativa está fuertemente relacionada con la posición del alumno en una distribución tradicional en filas (Marx et al., 2000). Investigaciones del aula tradicional con alumnos sentados en filas enfrentados a la maestra han tendido a indicar que aquellos alumnos sentados en la parte delantera y central de la clase se comunican más con la maestra. En este sentido, MacPherson (1984) analiza el entorno del aula tradicional y señala que la percepción que los alumnos tienen sobre las ventajas y desventajas de estar localizados en la parte delantera del aula tiene que ver con la proximidad al maestro y el pizarrón como agentes educativos. Así mismo, los alumnos destacan la visibilidad del pizarrón, la audibilidad del maestro y la oportunidad de interacción uno a uno como aspectos destacables de estar ubicados en la primera fila. Sommer (2007), por su parte, manifiesta que los alumnos ubicados en las primeras filas y en el centro del aula tienen mayor participación que aquellos sentados en los laterales. Por su parte, Lim y colegas (2012) señalan la existencia de espacios en el aula que adquieren significados específicos dependiendo del posicionamiento y la distancia de esta área en relación con el alumno y la maestra. En su trabajo investigan los diferentes tipos de espacios en el aula y el significado afectivo que se les asocia.

Es a partir de estos trabajos de investigación que este estudio encuentra su punto de partida. 
Se argumenta, por tanto, que la desatención de la perspectiva del alumno implica que partes de la lógica que estructuran la distribución espacial del aula quedan ignorados o descuidados. De hecho, a pesar de numerosos estudios, son pocos los investigadores que ponen la dimensión arquitectónica del aula bajo la lupa para determinar la relación de interacción que existe entre ellos y las fuentes de información.

\section{ESTRATEGIA METODOLÓGICA}

La estrategia metodológica que se propone es de corte fenomenológico ya que se argumenta que el problema arquitectónico no abarca tan solo lo construido sino que, también, a los individuos que acoge. De hecho, es la relación obra-individuo la que permite hablar, en términos contemporáneos, de fenómeno arquitectónico. En este sentido, se cuestiona la noción unilateral de 'hacer' arquitectura ya que los individuos juegan un papel fundamental en la dialéctica espacio - usuario.

Aceptando que todo tipo de registro de una experiencia es una reducción de la misma, la estrategia metodológica que se propone deriva básicamente (1) de estudios visuales (2) de las distancias derivadas de la proxémia y (3) de información video-fotográfica obtenida in situ, que se obtienen desde el punto de vista del alumno como usuario del espacio de aula.

\section{RELACIÓN VISUAL}

La visual de carácter experiencial se puede describir como el campo visual efectivo, fotográficamente abstraído, que se obtiene desde una ubicación determinada en el espacio. Esta visual plasma, de forma tangible y efectiva, lo que se ve del espacio en un instante desde una ubicación determinada. Es decir, que se busca ver lo que el alumno está viendo. Para este estudio, la visual del alumno se obtiene a través de la captura fotográfica que el mismo realiza desde su ubicación espacial en dirección al pizarrón. Esta imagen fotográfica proporciona datos concretos sobre los elementos que componen el campo visual del participante de donde se extraen los porcentajes de maestra, pizarrón y alumnos (ver figura 1).

\section{RELACIÓN PROXÉMICA}

Partiendo de la teoría de la proxemia, el concepto de esferas de interacción surge de las distancias de comunicación que define Hall (1969) como: íntima, personal, social y pública. Cada una de estas distancias, que se generan cuando dos o más personas interactúan, tiene características propias referidas al tipo de comunicación cara a cara que se lleva a cabo. A los efectos de este estudio, estas esferas comunicacionales sirven para estimar el tipo de relación de interacción que cada alumno mantiene con la maestra dentro del aula, siempre considerando que la ubicación del alumno en el aula es constante. Para llevar a cabo el registro se superponen las esferas - íntima, personal, social y pública - a la planta arquitectónica del aula de acuerdo con 


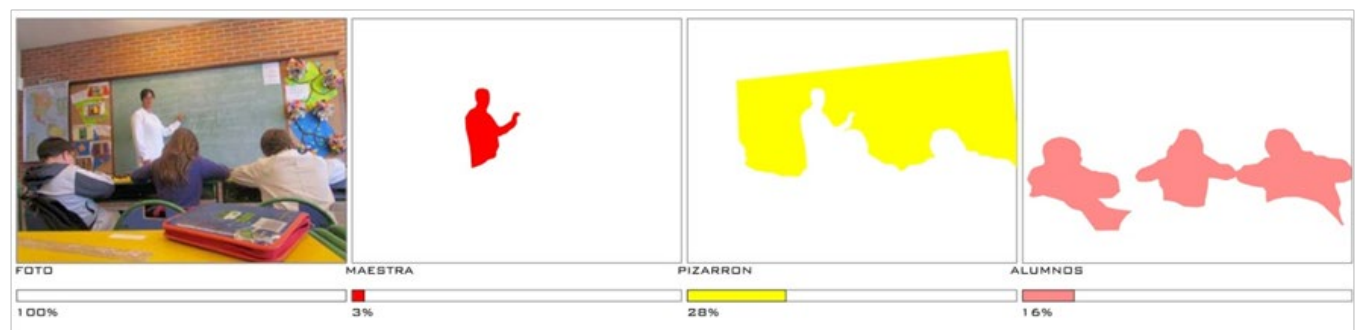

Figura 01.

Registro fotográfico de un alumno y la distinción de porcentajes de maestra, pizarrón y alumnos (elaboración propia).

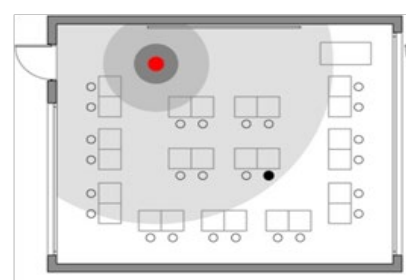

Estrerasgoial

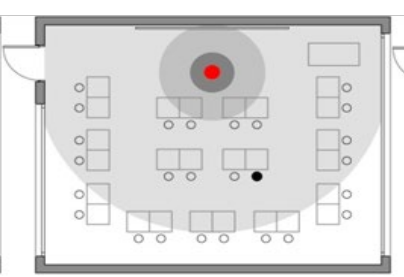

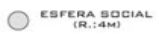

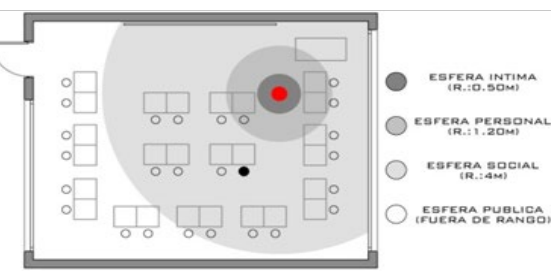

EsFera social

Figura 02.

Planta de aula con distancias proxémicas correspondiente a un alumno (elaboración propia).

la ubicación espacial de la maestra (figura 2). A los efectos de esta investigación, se realizan tres registros distintos cuya variación se vincula con las posiciones espaciales más comunes de la maestra. De este modo, las esferas se dibujan como arcos concéntricos a la maestra y plasman de forma gráfica la esfera en la cual queda ubicado el alumno. El siguiente ejemplo representativo muestra al alumno con un punto negro y a la maestra con un punto rojo. Para cada instancia de ubicación de la maestra, el alumno queda ubicado en una esfera que está determinada por la distancia que lo separa de la maestra.

\section{RELACIÓN CINÉIICA}

El registro del movimiento proporciona datos sobre los patrones de desplazamiento de las personas. El rastreo, entonces, implica el dibujo de líneas en un plano de acuerdo con el movimiento de las personas durante un período de tiempo determinado. Para el registro cinético dentro del aula, primeramente, se filma la clase donde la filmadora se coloca en un lugar determinado del aula desde donde se puede captar la mayor cantidad de actividad. La filmación se realiza con el fin de registrar los desplazamientos que realizan los alumnos y la maestra dentro del aula durante una hora de clase. A partir del video se extraen los desplazamientos de cada uno de los alumnos, 


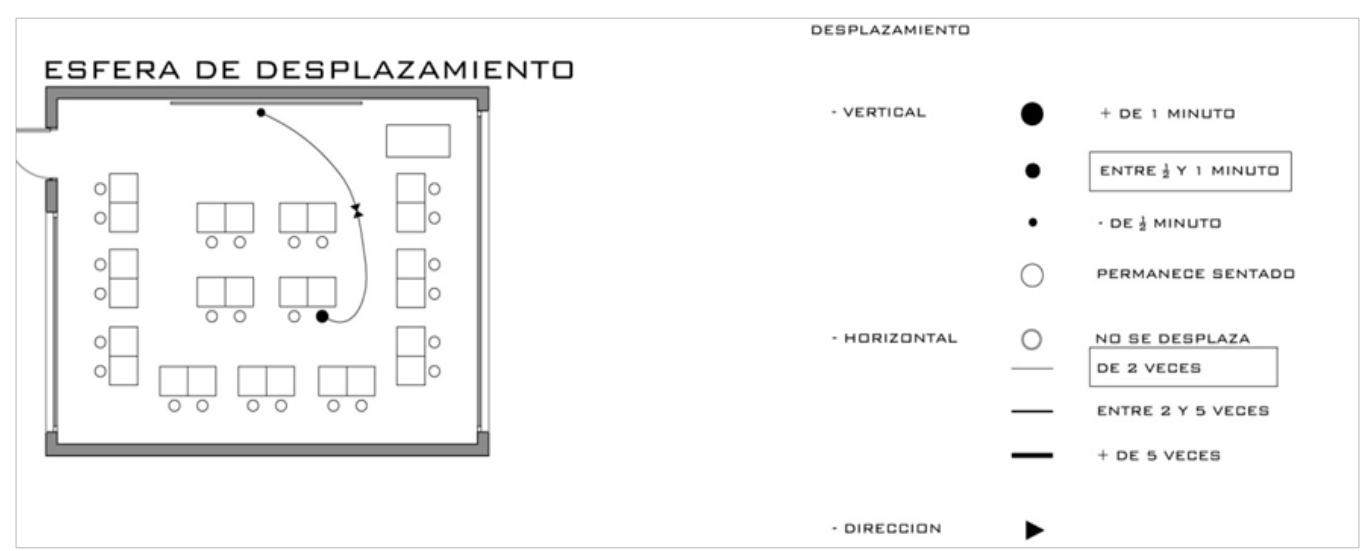

Figura 03.

Registro de la relación cinética de un alumno (elaboración propia).

AULA 1

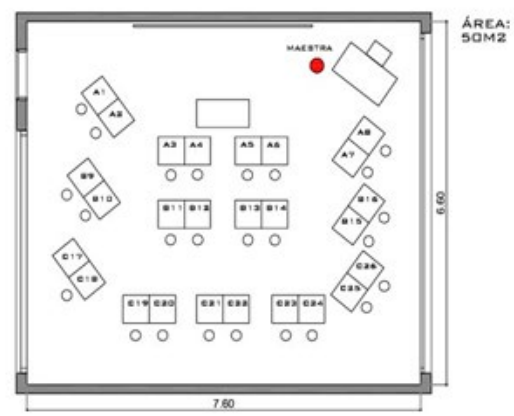

AULA 3

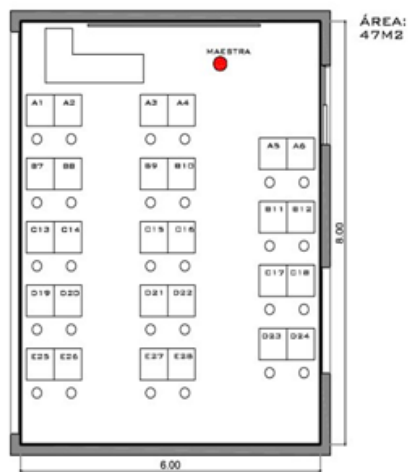

AULA 2

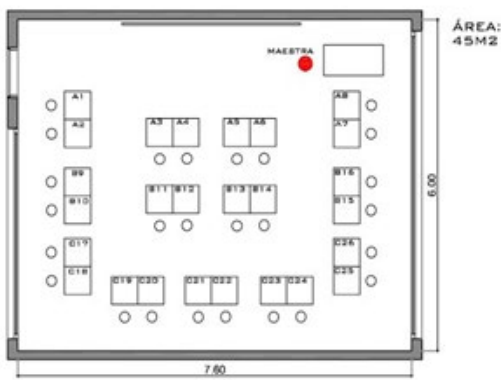

AULA 4

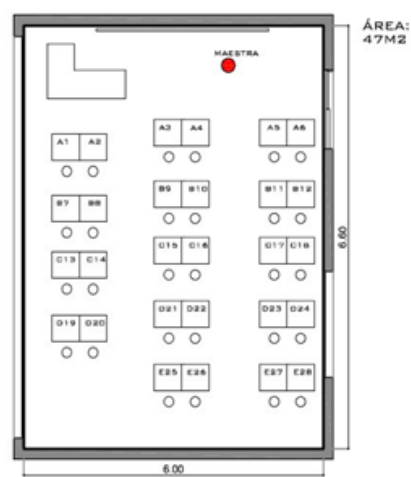

Figura 04.

Área, proporción y disposición espacial de las aulas de los casos de estudio. 
los cuales se dibujan como líneas en la planta arquitectónica el aula (figura 3).

Por último, se realizaron entrevistas con las maestras que informan sobre su percepción de lo que sucede en el espacio de aula. Las mismas se llevan a cabo con tres maestras que utilizan el método tradicional de enseñanza.

Los casos de estudio donde se realiza el análisis son cuatro aulas tradicionales ubicadas en dos escuelas en Uruguay. Las aulas albergan alumnos de 4to grado y acogen, respectivamente, 28 alumnos con 1 maestro a cargo. Su forma es rectangular y con una disposición tradicional de alumnos (figura 4).

\section{ANÁLISIS DE LOS CASOS DE ESTUDIO}

Del análisis vivencial de estos casos de estudio se identifican y cuantifican variaciones perceptivas de interacción entre alumno y maestro según relaciones de proporción y de disposición espacial.

\section{PROPORCIÓN DEL AULA}

Las variaciones en la proporción de aula permiten el aumento, o la disminución, de la cantidad de alumnos en relación visual óptima. En este sentido, se considera como relación 'óptima' cuando el alumno puede interaccionar con la fuente de información de forma fluida y sin mayores interferencias.

Las aulas 1 y 2 tienen proporción tendiente a la forma cuadrada (con proporción aproximada de 0,9$)$ donde la distancia máxima entre la ubicación del pizarrón y la pared del fondo es de 6 metros. Esto hace que el máximo de filas quede restringido y determinado por las proporciones del espacio. Implica, a su vez, que no exista la posibilidad de una distancia mayor a 6 metros entre la maestra y el alumno sentado en la fila del fondo. Por el contrario, las aulas 3 y 4 tienen forma rectangular, donde la profundidad del aula corresponde al lado más largo (de 8 metros). Esto hace que la distancia entre la maestra y los alumnos sentados al fondo del aula sea mayor y que, por tanto, el número de filas se pueda incrementar a cinco.

Las aulas 1 y 2, en este respecto, son $25 \%$ menos profundas comparadas con las aulas 3 y 4. Esto implica que en las dos primeras la distribución espacial sea en 3 filas, o sea que no existen alumnos en estas clases que tengan más de 2 alumnos por delante. Para los casos de aula 3 y 4 , por el contrario, la distribución espacial está constituida por 5 filas, que se distribuyen en profundidad, aspecto que implica que hay alumnos que tienen hasta 3 o 4 alumnos por delante.

Con respecto a la profundidad del aula, aquellos alumnos en la primera fila poseen los porcentajes de maestra y pizarrón más altos comparado con los alumnos localizados al fondo. Este porcentaje disminuye considerablemente en aquellos alumnos localizados en las filas posteriores donde para aquellos alumnos en la fila 2 se reduce un $50 \%$ y en la fila 3 la reducción 
es de un $75 \%$ de visual. Esta disminución abrupta en los porcentajes de los alumnos en la fila 3 corresponde a una distancia de 5 metros de los centros de información. Esto hace suponer que la profundidad de aula es un factor clave ya que un aula considerada profunda "invita" a tener mayor número de filas y que, por tanto, las proporciones del espacio ejercen un control directo en este sentido.

Las maestras, a su vez, coinciden en que la profundidad del aula está relacionada con sus posibilidades de interacción con los alumnos y señalan que los espacios de aula 'son rectángulos de un largo muy profundo, no es cuadrado o un rectángulo más cerca al cuadrado, es muy alargado, es muy larga la profundidad`' (Maestra, aula B).

Así mismo, se desprende del análisis proxémico que la cantidad de alumnos a una distancia recomendable (dentro de la esfera social proxémica) en las aulas 1 y 2 . En las aulas 3 y 4 hay 18 alumnos en cada aula que quedan en la esfera pública con respecto a la maestra. La cantidad de alumnos dentro de esta esfera en las aulas 1 y 2 desciende a la mitad, aspecto que se correlaciona con los resultados de la relación visual y las entrevistas e indican una vinculación directa con la profundidad del espacio de aula.

Los resultados del análisis permiten argumentar que la proporción de ancho de aula, a su vez, tiene influencia en la cantidad de alumnos en óptima relación visual. En este sentido, se constata que aquellos alumnos localizados en las zonas del costado del aula muestran bajos porcentajes de maestra y pizarrón en su campo visual aunque estén localizados en las zonas delanteras. La captura que efectúan los alumnos desde su ubicación en el espacio de aula muestra que aquellos ubicados a los costados del aula tienen visuales muy laterales de la maestra y el pizarrón. Este aspecto indica que quedan fuera del foco de atención de la clase con respecto a la maestra y sugiere una relación entre la percepción y la perspectiva de los alumnos.

La proporción del aula, a su vez, tiene incidencia en la cantidad de alumnos que puede alojar el espacio de aula ya que tener 28 alumnos dentro del aula hace que algunos queden en condiciones deterioradas con respecto a la interacción con la maestra. Los resultados obtenidos sugieren que el número de alumnos no debe superar los 24.. Así mismo, este aspecto encuentra su correlato en las entrevistas con las maestras quienes indican conciencia sobre la problemática del elevado número de alumnos en el aula. Específicamente, una de las maestras señala que:

- Cuando faltan a la clase te das cuenta, el espacio se agranda... son muchas horas y muchos nenes, mucha gente dentro de una clase` (Maestra, aula C).

A partir del análisis de los datos que surgen de los registros y de las entrevistas con las 
maestras, se puede intuir que para igualar las condiciones de interacción educativa, las proporciones del espacio deben estar acotadas no solo en la relación de profundidad sino que, también, en el ancho del espacio de aula.

\section{DISPOSICIÓN ESPACIAL DE LOS ALUMNOS}

La cantidad de filas dentro del aula impacta en el número de alumnos en óptima relación visual con la maestra y el pizarrón. Con la disposición espacial de las aulas 1 y 2 una mayor cantidad de alumnos interactúan de forma óptima con la maestra a través de la disposición en tres filas. Efectivamente, durante las entrevistas las maestras afirman que tres filas es lo más recomendable para este tipo de aulas ya que: Los que estaban atrás no estaban lejos (con la nueva disposición). Me parece que aunque fueran tres filas estaba perfecto. 0 sea, les veía las caras [...] cuando ya tenés 4 o 5 filas de alumnos con 3, 4 o 5 niños adelante no los ves" (Maestra, aula C).

A su vez, y tomando en cuenta la ideología de panóptico característico de la educación tradicional (Focault, 2002), las maestras indican la necesidad de estar en 'control de lo que sucede` con los alumnos dentro del aula. Así mismo señalan que ' ... en realidad es mejor tenerlos 'más cerca, más a mano' y 'mis ojos alcanzan a ver a todos por igual' (Maestra, aula C).

\section{CONCLUSIONES}

Existen muchas condicionantes físico-sociales con variadas connotación que infieren en la interacción alumno - maestro dentro del espacio de aula. En este estudio se extrajeron aspectos espaciales que se considera han estado relativamente postergados y que permiten una mejor comprensión del espacio físico de aula, sin olvidar el aspecto social ya que ambos son los que generan un ambiente óptimo para la interacción.

La metodología aplicada en los casos de estudio se basa, principalmente, en el análisis desde el punto de vista del alumno la interacción -con los centros de información (maestro y pizarrón) en el espacio. Este análisis de corte fenoménico permite la caracterización y cuantificación de características arquitectónicas del aula que no habían sido previamente analizadas desde un punto de vista vivencial y por tanto serán de utilidad para informar diseños futuros.

Los resultados muestran que a partir de una distancia de 5 metros desde la maestra el deterioro acumulado de interacción de los alumnos cae abruptamente y es superior al 50\% comparado con los restantes; aspecto que se hace más severo según la relación longitudinal del aula. La interacción queda entonces afectada por la proporción del aula, a partir de donde se puede estimar que la situación recomendada tendería a una proporción similar de ancho y profundidad del espacio. 
Más allá del potencial analítico del análisis vivencial, es más importante aun la posibilidad de potenciar el diseño informado en base a evidencia de nuevas configuraciones espaciales. Es fundamental destacar, entonces, que la posibilidad de trasformar el concepto de análisis vivencial en una herramienta de diseño implica un cambio en el acto de diseñar. Este nuevo método sugerido puede ayudar a los arquitectos a explorar formas, proporciones y distribuciones espaciales más complejas y eficientes otorgando seguridad a la hora de proponer aproximaciones al problema en las diferentes etapas del diseño proyectual. Pues, sea cual sea la forma, el objetivo central de los análisis perceptivo/vivencial es optimizar y equilibrar la interacción entre los usuarios durante la utilización de los espacios educativos, a la vez de abrir nuevas líneas de investigación, diseño y de cómo pensar el espacio arquitectónico. 


\section{BIBLIOGRAFÍA}

AMRINE, F., ZUCKER, F. y WHEELER, H. (1987). Goethe and the sciences: A reappraisal. Boston: Kluwer Academic Publishers.

AMRINE, F.(1995). Goethe in the history of science. New York: Grove/Atlantic.

BARRAN, P. (2008). Interacciones entre las prácticas proyectuales y las ideas educativas en el Uruguay moderno y contemporáneo. Montevideo: FARQ - UdelaR

BARRETT, P., DAVIES, F., ZHANG, Y. y BARRETT, L. (2015). The impact of classroom design on pupils' learning: Final results of a holistic, multi-level analysis. Building and Environment, 89 (Julio), 118-133.

BRAILOVSKY, D. (2012). La Escuela Y Las Cosas. Experiencia escolar a través de los objetos. Rosario: Homo Sapiens Ediciones.

BURGOS, F. (2001). Revolución en las aulas. Arquitectura Viva, 78, 17 - 22.

BURKE, C. y GROSVENOR, I. (2008) School. London: Reaktion Books.

CARDELLINO, P.; VARGAS SOTO, E. Y ARANEDA C. (2017). La evolución del diseño de aula escolar: los casos de Uruguay y Costa Rica [en línea] Fecha de consulta: 23-08-18. ACE: Architecture, City and Environment $=$ Arquitectura, Ciudad y Entorno, 12(34), 97122.

CARUSO, M., y DUSSEL, I. (1999). La invención del aula. Una genealogía de las formas de enseñar. Buenos Aires: Santillana.
CORONA MARTÍNEZ, A. (2009) Ensayo sobre el proyecto. Buenos Aires: Nobuko.

DARMODY, M. y SMYTH, E. (2012) Exploring school and classroom environments in Irish primary schools. Children, Youth and Environments, 22(1), 178-197.

DAVIS, F. (1978) La comunicación no verbal, $7^{\text {a }}$ edición. Madrid: Alianza.

FOUCAULT, M. (2002) Vigilar y castigar : nacimiento de la prisión.- 1a, edición. Buenos Aires : Siglo XXI Editores.

FRAGOSO FRANCO, D. (1999) La comunicación en el salón de clases. Razón y palabra, 13(4).

GARCÍA PONCE, I. (2000) Cinética y proxemia en el aula. Educacion, IX, 18 (Septiembre).

GEHL, J. y SVARRE, B. (2013) How to study public life. Washington: Island Press.

GISLASON, N. (2007). Placing education: The school as architectural space. Paideusis, 16(3), 5-14.

GREMENTIERI, F., y SHMIDT, C. (2010). Arquitectura, educación y patrimonio: Argentina 1600-1975. Buenos Aires: Pamplatina.

GUMP, P. (1987). School and classroom environments. In I. Altman, And J.F.Wohlwill (eds), Handbook of environmental psychology (pp. 131-174). New York: Plenum Press. 
HEIDEGGER, M. (1962) Being and Time. New York: Harper \& Row.

PIÑON, H. (2006) Teoría del proyecto. Barcelona: Edicions de la Universitat Politècnica de Catalunya.

HERTZBERGER, H. (2008) Space and learning. Rotterdam: 010 Publishers.

HIGGINS, S., HALL, E., WALL, K., WOOLNER, P. y MCCAUGHEY, C. (2005). The Impact of School Environments: A Literature Review, London: Design Council.

HALL, E. (1969) The Hidden Dimension. Nueva York: Anchor Books Editions.

HOLLIMAN, W.B. y ANDERSON, H.N. (1986). Proximity and student density as ecological variables in a college classroom. Teaching of Psychology, 13 (4), 200-203.

HORNE-MARTIN, S. (2004) Environmentbehaviour studies in the classroom. The Journal of Design and Technology Education: 9(2), 77-89.

LAWSON, B. (2001) The language of space. Oxford: Architectural Press.

LEIRINGER, R. y CARDELLINO, P. (2011) Schools for the twenty-first century: school design and educational transformation. British Educational Research Journal, 37(6), 915934.

LIM, F. V., O'HALLORAN, K. L., PODLASOV, A. (2012) Spatial pedagogy: mapping meanings in the use of classroom space. Cambridge Journal of Education, 42(2), 235 - 251.
MACPHERSON, J. C. (1984). Environments and interaction in row-and-column classrooms, Environment and Behavior, 16(4): 481-502.

MARX, A, FUHRER, U. y HARTIG, T. (2000). Effects of classroom seating arrangements on children's question-asking. Learning Environments Research, 2 (3), 249-263.

MUÑOZ COSME, A. (2008) El proyecto de arquitectura: concepto, proceso y representación. Barcelona: Reverté.

SCOTT-WEBBER, L., BRANCH, J., BARTHOLOMEW, P. y NYGAARD, C. (2014) Practising Learning Space Design. En: Nygaard, C., Branch, J., Scott-Webber, L. y Bartholomew, P. (eds) Learning Space Design in Higher Education. Faringdon: Libri Publishing, pp. 1-20.

SEAMON, D. (1990) Awareness and reunion: A phenomenology of the person-environment relationship as portrayed in the New York photographs of André Kertész. En: ZONN, L. (ed.) Place images in the media. New Jersey: Totowa, Roman and Littlefield, pp. 87-107.

SEAMON, D. (2000) A way of seeing people and place: Phenomenology in environmentbehavior research. En: WAPNER, S.; DEMICK, J., YAMAMOTO, T. y MINAMI, H. (eds) Theoretical perspectives in EnvironmentBehavior Research. New York: Plenum, pp. 157 - 178.

SEAMON, D. y ZAJONC, A. (1998) Goethe's Way of Science: A phenomenology of nature. New York: SUNY Press.

SOMMER, R. (2007) Personal space. The behavioral basis of design. Edición actualizada. Bristol: Bosko Books. 
STEINER, R. (1989). Goethe y su visión del mundo. Madrid: Editorial Rudolf Steiner.

STEINER, R. (2000) Nature's Open Secret: Introductions to Goethe's Scientific Writings. New York: Anthroposophic Press.

STEINER, R. (2008) Goethe's theory of knowledge.An outline of the epistemology of his worldview. New York: Anthroposophic Press.

STEWART, D. y MUKUNAS, A. (1990) Exploring phenomenology: A guide to the field and its literature. 2da edición. Ohio: Ohio University Press.

VARELA, F. THOMSON, E. y ROSCH, E. (2009) De cuerpo presente: Las ciencias cognitivas y la experiencia humana. 4ta. Edición. Barcelona: Editorial Gedisa.

WOOLNER, P. (2010) The Design of Learning Spaces. London: Continuum.

WOOLNER, P. (2015) School Design Together. London: Routledge.

ZAJONC, A. (1994) Atrapando la luz. Santiago: Editorial Andrés Bello. 
